\title{
DISTRIBUCIÓN DE LOS URÓLOGOS EN ESPAÑA: SITUACIÓN ACTUAL Y ESTIMACIÓN DE NECESIDADES FUTURAS MEDIANTE UN MODELO PREDICTIVO
}

\author{
P. LÁZARO Y DE MERCADO ${ }^{1}$, A. ALLONA ALMAGRO ${ }^{2}$, O. LEIVA GALVIS ${ }^{3}$ \\ ${ }^{1}$ Investigador. Técnicas Avanzadas de Investigación en Servicios de Salud. Madrid. ${ }^{2}$ Secretario. AEU. \\ ${ }^{3}$ Presidente. AEU. \\ Actas Urol Esp. 27 (8): 569-580, 2003
}

\section{INTRODUCCIÓN}

Desde que hace unos 100 años, la Urología se individualizó de la Cirugía, se ha consolidado como especialidad por la relevancia de las patologías que aborda y por nuevas técnicas específicas como la urografía, la endoscopia, los trasplantes renales o la litotricia, entre otros. En España, la Urologia ha experimentado importantes cambios a lo largo de los últimos años, y es de esperar que continúe este proceso en el futuro previsible. La innovación tecnológica, los avances científicos, los cambios organizativos en el Sistema Nacional de la Salud, los cambios sociales, y los cambios demográficos, de alguna manera afectarán la organización de la asistencia urológica, la demanda asistencial, incluso la prevalencia de algunas enfermedades urológicas. La oferta de servicios asistenciales también puede ser cambiante en función de los urólogos que se jubilan, residentes que se forman, servicios que se crean, $y$ otros factores. La inadecuación de la oferta a la demanda puede crear desajustes, tanto en el ámbito nacional como regional. Los desajustes pueden dar lugar a tiempos de espera inaceptables, variabilidad en la carga de trabajo, variabilidad en la calidad, desigualdad en el acceso, o ineficiencia en la prestación de la atención urológica.

Con estos antecedentes, la Asociación Española de Urología (AEU) se formuló diversas preguntas centradas en el número de urólogos que realmente hay en España, y en estimar los urólogos que podrían ser necesarios en el futuro. Para responder a estas cuestiones, la AEU encargó un estudio a Técnicas Avanzadas de Investigación en
Servicios de Salud (TAISS) en Julio de 2002. Abbott Laboratories S.A. (Abbott), patrono de la AEU, patrocinó el estudio. En este artículo se presenta un resumen del estudio y sus hallazgos fundamentales. En un futuro próximo se publicará el estudio completo en distintos formatos, incluidas versiones accesibles en la página web de la AEU.

\section{OBJ ETIVOS}

1. Describir la distribución de los urólogos en España en función de sus características profesionales y sociodemográficas.

2. Identificar las variables fundamentales demográficas y de prevalencia de las que dependa la carga de trabajo en urología en España para los próximos 10 años.

3. Construir un modelo predictivo sobre la necesidad de urólogos en España para los próximos 10 años basado en los hallazgos de los objetivos anteriores.

\section{METODOLOGÍA}

Objetivo 1. Describir la distribución los médicos disponibles en la especialidad de Urología en España en función de sus características profesionales y sociodemográficas. Para desarrollar este objetivo se han desarrollado diversas tareas.

Definición de variables. La dirección de la AEU decidió conocer de cada urólogo las siguientes variables: edad, sexo, especialidad, sector (público/privado), categoría profesional, subespecialidad, y provincia. Para recoger las variables de interés se elaboró un cuestionario probado en un estudio piloto. 
Identificación de urólogos. Para identificar a los urólogos existentes en España, se ha partido de tres fuentes de datos: 1) la base de datos de socios de la AEU y de ENE ediciones; 2) centros hospitalarios donde puede haber recursos humanos de urología; y 3) cuadros médicos de compañias aseguradoras privadas.

1. Identificación de urólogos socios de la AEU. Creación de la base de datos BDOU-1. Mediante la fusión de las bases de datos de la AEU y de ENE ediciones, se han identificado 1.632 urólogos españoles no jubilados. Esta base de datos se llama BDOU-1 (primera base de datos operativa de urólogos) y en ella constan las variables nombre, apellidos, y dirección. A estos urólogos se les envió el cuestionario por correo postal en tres oleadas sucesivas. A 11 de abril de 2003, habían respondido 1.102 (67,5\%).

\section{Identificación de urólogos en servicios de} Urología. Creación de la base de datos BDOU-2. Se identificaron 326 centros sanitarios con servicios o secciones de Urología. A los responsables de estas unidades se les envió un cuestionario para que facilitasen la lista de sus urólogos, con su categoría profesional. Este envío se hizo en dos oleadas, la primera el 2 de septiembre, y la segunda el 15 de noviembre de 2002. Ambas oleadas se hicieron por dos vías simultáneas: correo postal y distribución por los delegados de Abbott. Respondieron 222 (68\%) jefes de servicio, que aportaron datos de 1.533 urólogos, con los que se ha construido la base de datos BDOU-2. Esta base de datos ha identificado a 281 urólogos que no estaban en la BDOU-1, a los cuales se les envió el cuestionario por correo el día 30 de enero de 2003. A fecha 11 de abril, habían respondido 84 (30\%).

3. Identificación de urólogos del sector privado. Creación de la base de datos BDOU-3. Se ha identificado a todos los urólogos que forman parte del cuadro médico de Asisa, Adeslas, MapfreCajaSalud, Sanitas y DKV Seguros, mediante búsquedas en las páginas web de las mencionadas compañías. Se obtuvieron 3.091 registros en el ámbito de las 50 provincias españolas. Una vez eliminadas las redundancias, esta base de datos ha permitido identificar a 1,267 urólogos, de los cuales, 120 no constaban en la BDOU-1 ni en la BDOU-2. A estos 120 urólogos, se les envió el cuestionario por correo el día 30 de enero de 2003. A 11 de abril, habían respondido 32 (27\%).

Identificación completa de urólogos. Creación de la base de datos definitiva (BDDU). Con la información original de las bases de datos operativas se diseñó y alimentó la base de datos definitiva de urólogos (BDDU), que finalmente contiene 2.033 urólogos activos. Esta base se completó con las respuestas de los 1.218 urólogos activos que han respondido al cuestionario, y es la base definitiva para los análisis y para el modelo predictivo.

Objetivo 2. Identificar las variables fundamentales demográficas y de prevalencia de las que dependa la carga de trabajo en urología en España para los próximos 10 años.

Se han obtenido datos del Instituto Nacional de Estadística (INE) sobre la población española hasta el año 2025 para el conjunto nacional, y por grupos de edad y sexo. Por esta razón, se ha extendido el horizonte temporal de aplicabilidad del modelo hasta el año 2025, en lugar de los 10 años previstos en el objetivo 2. Sin embargo, por comunidades autónomas (CCAA) el INE sólo ha calculado predicciones hasta el año 2005. Para disponer de las proyecciones para todos los años, TAISS ha calculado la población por CCAA, edad, y sexo, hasta el año 2025 ponderando la población nacional según la población de la CCAA del último año proyectado por el INE. Para estimar el peso asistencial que tienen determinadas enfermedades urológicas, se ha asignado a la población una cifra de incidencia de cáncer de vejiga, cáncer de próstata, y cáncer de riñón, y de prevalencia de hipertrofia benigna de próstata, incontinencia urinaria, litiasis renal, y disfunción eréctil, cada una de ellas a su grupo de edad y sexo correspondiente. Estas cifras de incidencia o prevalencia de las condiciones elegidas se han buscado en diversas fuentes bibliográficas ${ }^{1-30}$, entre las cuales se han seleccionado las más verosimiles para España. Finalmente, se ha estimado la carga de trabajo anual de las patologías urológicas seleccionadas como porcentaje de toda la actividad de un servicio de urología típico. 
Objetivo 3. Construir un modelo predictivo sobre la necesidad de urólogos en España para los próximos 10 años.

Se ha construido un modelo predictivo cuyo motor de cálculo se basa en información sobre la oferta (obtenida en el objetivo 1), la demanda (obtenida en el objetivo 2), las asunciones, y los criterios usados para adaptar la oferta a la demanda. Las asunciones consisten en valores aplicados cuando la variable no es conocida. Por ejemplo, el número de residentes que se formará en el año 2006, cambios de incidencia de algunas patologias, o efectos de nuevas tecnologias. Los criterios consisten en la definición explícita de un indicador que se quiera cumplir. Por ejemplo, el número de urólogos deseado por millón de habitantes en el año 2010. Combinando matemáticamente la oferta, demanda, asunciones, y criterios, el modelo identifica los urólogos necesarios en el año que se desee y los que deben producirse cada año hasta el año deseado. $\mathrm{El}$ modelo permite efectuar predicciones hasta el año 2025, para el conjunto nacional y para cada una de las 17 CCAA. La interfaz del modelo en la pantalla de un ordenador se muestra en la Figura 1.

\section{RESULTADOS}

La población de urólogos actual.

Con la estrategia utilizada en este proyecto se han identificado 2.033 urólogos activos (no jubilados) en España. De todos ellos se conoce la provincia en la que trabaja y el sexo. Otras variables se conocen en la medida que esta información exista en la base de datos original o si el urólogo ha contestado al cuestionario. De los 2.033 urólogos activos identificados, respondieron a la encuesta 1.218 (tasa de respuesta del 59,9\%).

La edad media de los urólogos españoles es de 46,4 años ( $\mathrm{DE}=10,2$ años), el $28 \%$ tienen menos de 40 años, el $54 \%$ tienen entre 40 y 55 años, un $13 \%$ tiene entre 56 y 65 años, y un 5\%, aún estando activo, tiene más de 65 años (Tabla I). El $92,4 \%$ de los urólogos son varones, el $82,9 \%$ trabaja en el sector público, el 53,6\% en el privado, y el $36,5 \%$ trabajan en ambos sectores. De los 652 urólogos que han informado sobre su especialidad, la mitad declaran dedicarse a la oncología, casi un $40 \%$ a la endoscopia o andrología, un $28 \%$ a la litiasis, una cuarta parte a la urodinámica, un $20 \%$ al trasplante renal, y un $13 \%$ a la

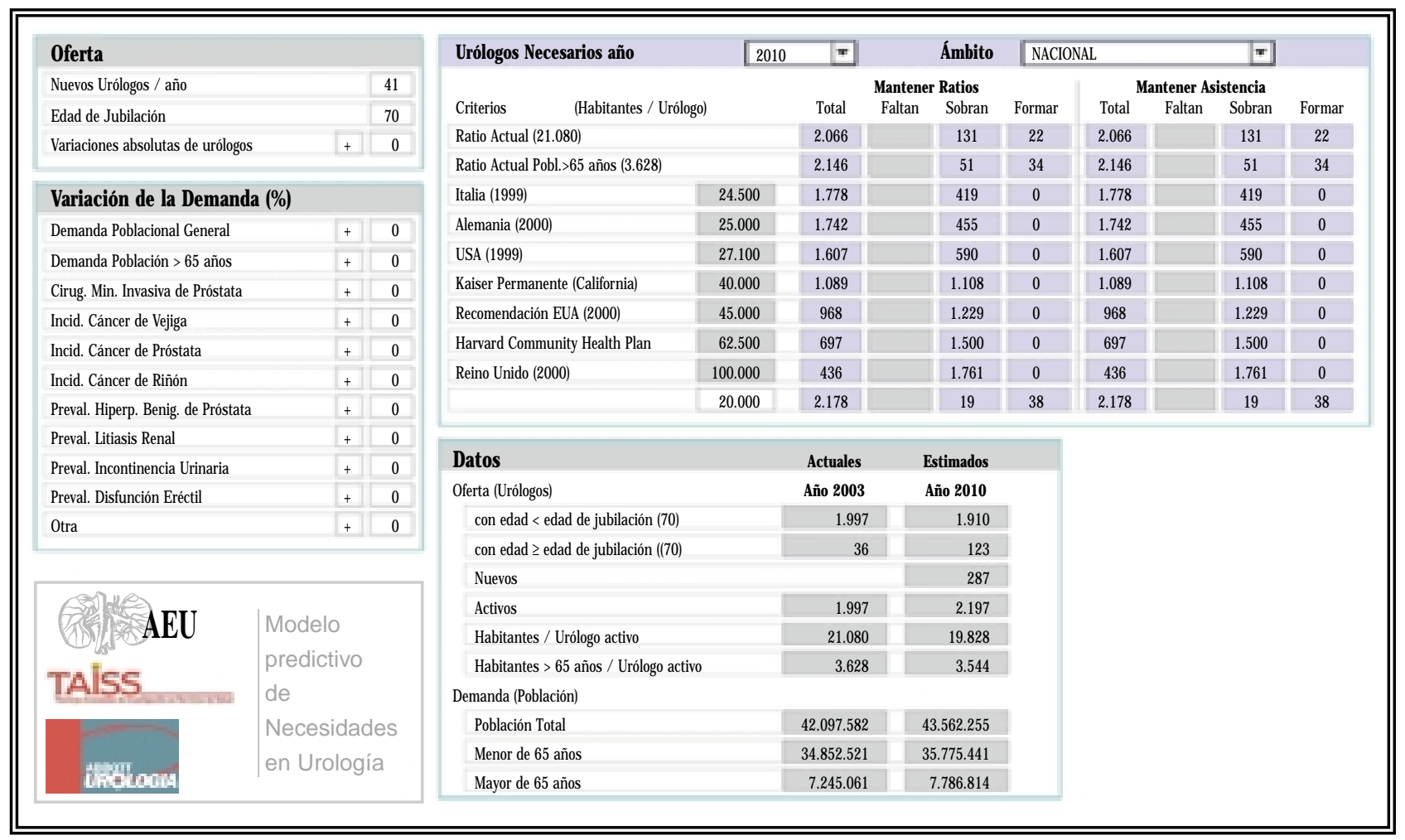

FIGURA 1. Interfaz del modelo predictivo en la pantalla del ordenador. 
TABLA I

ANÁLISIS DESCRIPTIVO DE LOS URÓLOGOS ESPAÑOLES EN EL AÑO 2003 (N=2.033)

\begin{tabular}{|c|c|c|c|}
\hline Variable & Categoría & $\mathbf{n}$ & $\%$ \\
\hline Edad $(\mathrm{N}=1.576)$ & $\begin{array}{l}<40 \text { años } \\
40-55 \text { años } \\
56-65 \text { años } \\
>65 \text { años }\end{array}$ & $\begin{array}{c}438 \\
858 \\
206 \\
74\end{array}$ & $\begin{array}{c}27,8 \\
54,4 \\
13,1 \\
4,7\end{array}$ \\
\hline Sexo $(N=2.033)$ & $\begin{array}{l}\text { Hombre } \\
\text { Mujer }\end{array}$ & $\begin{array}{c}1.879 \\
154\end{array}$ & $\begin{array}{c}92,4 \\
7,6\end{array}$ \\
\hline Sector $(\mathrm{N}=1.869)$ & $\begin{array}{l}\text { Público } \\
\text { Privado } \\
\text { Ambos }\end{array}$ & $\begin{array}{c}1.550 \\
1.002 \\
683\end{array}$ & $\begin{array}{l}82,9 \\
53,6 \\
36,5\end{array}$ \\
\hline Especialidad $(\mathrm{N}=652)$ & $\begin{array}{l}\text { Oncología } \\
\text { Endoscopia } \\
\text { Andrologia } \\
\text { Litiasis } \\
\text { Urodinámica } \\
\text { Trasplante renal } \\
\text { Urología pediátrica } \\
\text { Una } \\
\text { Dos } \\
\text { Tres } \\
\text { Cuatro } \\
\text { Cinco } \\
\text { Seis } \\
\text { Las siete }\end{array}$ & $\begin{array}{c}332 \\
244 \\
240 \\
179 \\
157 \\
129 \\
84 \\
\\
312 \\
152 \\
85 \\
51 \\
29 \\
16 \\
7\end{array}$ & $\begin{array}{r}50,9 \\
37,4 \\
36,8 \\
27,5 \\
24,1 \\
19,8 \\
12,9 \\
\\
47,9 \\
23,3 \\
13,0 \\
7,8 \\
4,4 \\
2,5 \\
1,1\end{array}$ \\
\hline Categoría $(\mathrm{N}=1.632)$ & $\begin{array}{l}\text { Jefe de Servicio } \\
\text { Jefe de Sección } \\
\text { Adjunto / FEA } \\
\text { Residente } \\
\text { Cupo } \\
\text { No jerarquizado }\end{array}$ & $\begin{array}{c}154 \\
197 \\
1.062 \\
202 \\
15 \\
2\end{array}$ & $\begin{array}{c}9,4 \\
12,1 \\
65,1 \\
12,4 \\
0,9 \\
0,1\end{array}$ \\
\hline Miembros AEU (N=2.033) & $\begin{array}{l}\text { Miembros } \\
\text { No miembros }\end{array}$ & $\begin{array}{c}1.632 \\
401\end{array}$ & $\begin{array}{l}80,3 \\
19,7\end{array}$ \\
\hline
\end{tabular}

urología pediátrica. Más de la mitad de los urólogos practican más de una de las subespecialidades mencionadas: el $23 \%$ practica dos de las especialidades, el $13 \%$ practica tres, y una proporción decreciente practica más de tres especialidades. La categoría profesional se ha podido establecer en 1.632 urólogos, de los cuales, el 9\% son jefes de servicio, el $12 \%$ jefes de sección, el $65 \%$ adjuntos, y el $12 \%$ residentes. De los 2.033 urólogos activos identificados, $1.632(80,3 \%)$ son miembros de la AEU.
En España, se dispone de 48,3 urólogos por millón de habitantes (20.707 habitantes por urólogo). Sin embargo, la distribución de urólogos por CCAA muestra una enorme variabilidad (Tabla II). Destacan por encima de la media Navarra con 71,4 urólogos por millón de habitantes (13.998 habitantes por urólogo), Aragón con 70,1 urólogos por millón de habitantes (14.267 habitantes por urólogo), y Madrid con 62,4 urólogos por millón de habitantes (16.031 habitantes por urólogo). Las CCAA menos dotadas son 
TABLA II

DISTRIBUCIÓN DE URÓLOGOS EN ESPAÑA POR COMUNIDADES AUTÓNOMAS EN EL AÑO 2003

\begin{tabular}{|c|c|c|c|c|}
\hline Comunidad Autónoma & Población* & Urólogos** & $\begin{array}{c}\text { Urólogos por millón } \\
\text { de habitantes }\end{array}$ & $\begin{array}{c}\text { Habitantes por } \\
\text { urólogo }\end{array}$ \\
\hline Andalucía & 7.514 .356 & 315 & 41,9 & 23.855 \\
\hline Aragón & 1.226 .936 & 86 & 70,1 & 14.267 \\
\hline Asturias & 1.081 .831 & 62 & 57,3 & 17.449 \\
\hline Baleares & 922.781 & 36 & 39,0 & 25.633 \\
\hline Canarias & 1.853 .105 & 65 & 35,1 & 28.509 \\
\hline Cantabria & 545.920 & 21 & 38,5 & 25.996 \\
\hline Castilla y León & 2.498 .892 & 120 & 48,0 & 20.824 \\
\hline Castilla-La Mancha & 1.793 .233 & 68 & 37,9 & 26.371 \\
\hline Cataluña & 6.551 .302 & 326 & 49,8 & 20.096 \\
\hline Extremadura & 1.079 .122 & 46 & 42,6 & 23.459 \\
\hline Galicia & 2.755 .042 & 114 & 41,4 & 24.167 \\
\hline Madrid & 5.562 .688 & 347 & 62,4 & 16.031 \\
\hline Murcia & 1.233 .088 & 52 & 42,2 & 23.713 \\
\hline Navarra & 573.903 & 41 & 71,4 & 13.998 \\
\hline País Vasco & 2.123 .310 & 92 & 43,3 & 23.079 \\
\hline Rioja & 283.621 & 11 & 38,8 & 25.784 \\
\hline Comunidad Valenciana & 4.352 .373 & 222 & 51,0 & 19.605 \\
\hline Ceuta & 76.542 & 6 & 78,4 & 12.757 \\
\hline Melilla & 69.537 & 3 & 43,1 & 23.179 \\
\hline Total Nacional & 42.097 .582 & 2.033 & 48,3 & 20.707 \\
\hline
\end{tabular}

*Cifras estimadas de población para el año 2003 a partir de las cifras oficiales de población a uno de enero de 2002 y proyecciones. Fuente: INE: www.ine.es (consultado el 7 de febrero de 2003).

**Datos de la encuesta a urólogos españoles a fecha de 11 de abril de 2003.

Canarias con 35, 1 urólogos por millón de habitantes (28.509 habitantes por urólogo), CastillaLa Mancha con 37,9 urólogos por millón de habitantes (26.371 habitantes por urólogo), y Cantabria con 38,5 urólogos por millón de habitantes (25.996 habitantes por urólogo). También por provincias existe una gran variabilidad (Tabla III). Destacan Soria, Ceuta, Zaragoza y Navarra con más de 70 urólogos por millón de habitantes (menos de 14.000 habitantes por urólogo) por encima de las demás provincias españolas. Las provincias con menos urólogos por población son Lugo, Jaén, y Orense con menos de 30 urólogos por millón de habitantes (más de 34.000 habitantes por urólogo).
Frecuencia de enfermedades urológicas relevantes y carga de trabajo asistencial.

Para los cálculos del modelo, se han asignado unas cifras de frecuencia de enfermedad consistentes con la bibliografía consultada. La prevalencia de hipertrofia benigna de próstata oscila entre el 0,6\% en varones entre los 40-49 años y el $30 \%$ en los mayores de 70 años ${ }^{1}$. La prevalencia de disfunción eréctil oscila entre el 3,9\% en los varones con 25 a 30 años, y el 32,3\% en los varones de 60 a 70 años $^{13}$. La prevalencia de incontinencia urinaria oscila entre el 9\% para los varones de 65 a 69 años, y el 35\% para las mujeres mayores de 85 años $^{20}$. La prevalencia de litiasis renal en los sujetos mayores de 25 años varía 
TABLA III

DISTRIBUCIÓN DE URÓLOGOS EN ESPAÑA POR PROVINCIAS Y COMUNIDADES AUTÓNOMAS

\begin{tabular}{|c|c|c|c|c|}
\hline $\begin{array}{l}\text { Comunidad Autónoma/ } \\
\text { Provincia }\end{array}$ & Población* & Urólogos** & $\begin{array}{l}\text { Urólogos por millón de } \\
\text { habitantes }\end{array}$ & $\begin{array}{c}\text { Habitantes por } \\
\text { urólogo }\end{array}$ \\
\hline Andalucía & 7.514 .356 & 315 & 41,9 & 23.855 \\
\hline Almería & 549.123 & 18 & 32,8 & 30.507 \\
\hline Cádiz & 1.146 .273 & 43 & 37,5 & 26.658 \\
\hline Córdoba & 774.835 & 28 & 36,1 & 27.673 \\
\hline Granada & 822.893 & 53 & 64,4 & 15.526 \\
\hline Huelva & 467.168 & 144 & 30,0 & 33.369 \\
\hline Jaén & 650.497 & 19 & 29,2 & 34.237 \\
\hline Málaga & 1.336 .399 & 56 & 41,9 & 23.864 \\
\hline Sevilla & 1.767 .168 & 84 & 47,5 & 21.038 \\
\hline Aragón & 1.226 .936 & 86 & 70,1 & 14.267 \\
\hline Huesca & 210.580 & 10 & 47,5 & 21.058 \\
\hline Teruel & 138.405 & 5 & 36,1 & 27.681 \\
\hline Zaragoza & 877.951 & 71 & 80,9 & 12.366 \\
\hline Asturias & 1.081 .831 & 62 & 57,3 & 17.449 \\
\hline Baleares & 922.781 & 36 & 39,0 & 25.633 \\
\hline Canarias & 1.853 .105 & 65 & 35,1 & 28.509 \\
\hline Las Palmas & 955.860 & 30 & 31,4 & 31.862 \\
\hline Santa Cruz de Tenerife & 897.245 & 35 & 39,0 & 25.636 \\
\hline Cantabria & 545.920 & 21 & 38,5 & 25.996 \\
\hline Castilla y León & 2.498 .892 & 120 & 48,0 & 20.824 \\
\hline Ávila & 166.371 & 6 & 36,1 & 27.729 \\
\hline Burgos & 355.357 & 16 & 45,0 & 22.210 \\
\hline León & 500.364 & 21 & 42,0 & 23.827 \\
\hline Palencia & 177.440 & 8 & 45,1 & 22.180 \\
\hline Salamanca & 349.712 & 17 & 48,6 & 20.571 \\
\hline Segovia & 150.401 & 6 & 39,9 & 25.067 \\
\hline Soria & 92.170 & 9 & 97,6 & 10.241 \\
\hline Valladolid & 504.900 & 29 & 57,4 & 17.410 \\
\hline Zamora & 202.177 & 8 & 39,6 & 25.272 \\
\hline Castilla-La Mancha & 1.793 .233 & 68 & 37,9 & 26.371 \\
\hline Albacete & 374.122 & 15 & 40,1 & 24.941 \\
\hline Ciudad Real & 487.381 & 19 & 39,0 & 25.652 \\
\hline Cuenca & 202.881 & 9 & 44,4 & 22.542 \\
\hline Guadalajara & 178.878 & 7 & 39,1 & 25.554 \\
\hline Toledo & 549.971 & 18 & 32,7 & 30.554 \\
\hline
\end{tabular}


TABLA III (Continuación)

DISTRIBUCIÓN DE URÓLOGOS EN ESPAÑA POR PROVINCIAS Y COMUNIDADES AUTÓNOMAS

\begin{tabular}{|c|c|c|c|c|}
\hline $\begin{array}{l}\text { Comunidad Autónoma/ } \\
\text { Provincia }\end{array}$ & Población* & Urólogos** & $\begin{array}{c}\text { Urólogos por millón de } \\
\text { habitantes }\end{array}$ & $\begin{array}{c}\text { Habitantes por } \\
\text { urólogo }\end{array}$ \\
\hline Cataluña & 6.551 .302 & 326 & 49,8 & 20.096 \\
\hline Barcelona & 4.939 .945 & 270 & 54,7 & 18.296 \\
\hline Gerona & 602.236 & 21 & 34,9 & 28.678 \\
\hline Lérida & 373.613 & 14 & 37,5 & 26.687 \\
\hline Tarragona & 635.508 & 21 & 33,0 & 30.262 \\
\hline Comunidad Valenciana & 4.352 .373 & 222 & 51,0 & 19.605 \\
\hline Alicante & 1.567 .210 & 58 & 37,0 & 27.021 \\
\hline Castellón & 504.210 & 26 & 51,6 & 19.393 \\
\hline Valencia & 2.280 .953 & 138 & 60,5 & 16,529 \\
\hline Extremadura & 1.079 .122 & 46 & 42,6 & 23.459 \\
\hline Badajoz & 666.559 & 29 & 43,5 & 22.985 \\
\hline Cáceres & 412.563 & 17 & 41,2 & 24.268 \\
\hline Galicia & 2.755 .042 & 114 & 41,4 & 24.167 \\
\hline Coruña & 1.119 .064 & 54 & 48,3 & 20.723 \\
\hline Lugo & 364.118 & 10 & 27,5 & 36.412 \\
\hline Orense & 345.987 & 10 & 28,9 & 34.599 \\
\hline Pontevedra & 925.873 & 40 & 43,2 & 23.147 \\
\hline Madrid & 5.562 .688 & 347 & 62,4 & 16.031 \\
\hline Murcia & 1.233 .088 & 52 & 42,2 & 23.713 \\
\hline Navarra & 573.903 & 41 & 71,4 & 13.998 \\
\hline País Vasco & 2.123 .310 & 92 & 43,3 & 23.079 \\
\hline Álava & 293.940 & 14 & 47,6 & 20.996 \\
\hline Guipúzcoa & 687.846 & 26 & 37,8 & 26.456 \\
\hline Vizcaya & 1.141 .524 & 52 & 45,6 & 21.952 \\
\hline La Rioja & 283.621 & 11 & 38,8 & 25.784 \\
\hline Ceuta & 76.542 & 6 & 78,4 & 12.757 \\
\hline Melilla & 69.537 & 3 & 43,1 & 23.179 \\
\hline Total nacional & 42.097 .582 & 2.033 & 48,3 & 20.707 \\
\hline
\end{tabular}

*Cifras estimadas de población a partir de las cifras oficiales de población a uno de enero de 2002 y proyecciones. Fuente INE: www.ine.es (consultado el 7 de febrero de 2003). **Datos de la encuesta a urólogos españoles a fecha de 11 de abril de 2003.

entre el 5,8\% para hombres y el 10\% para mujeres $^{28}$. Las incidencias brutas (casos por cada 100.000 habitantes) oscilan, en el cáncer de vejiga entre el 7,9 en mujeres y el 47,1 en hombres, en el cáncer de riñón entre el 6,1 en mujeres y el 14 en hombres, y en el cáncer de próstata es de $54,2^{30}$. En conjunto, las siete patologías elegidas suponen el $85 \%$ de la carga de trabajo en un servicio de urología español medio. La hipertrofia benigna de próstata supone el $27,5 \%$ del trabajo del servicio, el cáncer de vejiga el 18,5\%, la litiasis el 14,5\%, el cáncer de próstata el 13,8\%, la disfunción eréctil el 4,3\%, la incontinencia urinaria el 4\%, y el cáncer de riñón el 2,3\%. 
Predicciones sobre las necesidades de urólogos. Análisis de sensibilidad.

Aunque el modelo predictivo funciona para hacer predicciones hasta el año 2025 para el conjunto nacional y para cada una de las 17 CCAA, en este artículo utilizaremos como ejemplo las predicciones para el año 2010 para España, realizando un análisis de sensibilidad. En el análisis de sensibilidad se han construido tres escenarios: el caso basal (estimado más razonable), el caso más favorable (bajos estimados para las variables de demanda), y el caso más desfavorable (altos estimados para las variables de demanda). En los tres escenarios se ha asumido que se forman 41 residentes todos los años, que la edad de jubilación va a ser de 70 años, que no se irán urólogos españoles a trabajar a otros países, y que no vendrán urólogos de otros países a trabajar a España. En el modelo se pueden modificar todas estas asunciones, pero a efectos de este artículo, se han simplificado.

Para el caso basal, se ha considerado que el único aumento de demanda será el que dependa del aumento de la población. En este escenario, en el año 2010, 123 urólogos de la cohorte actual tendrán más de 70 años, se habrán formado mediante el programa MIR 287 urólogos nuevos, y por lo tanto habrá 2.197 urólogos activos. Esas cifras equivalen a tener un urólogo activo por cada 19.828 habitantes. Por lo tanto, si se quisieran mantener los ratios actuales por habitante sobrarian 131 urólogos, y en consecuencia habría que formar cada año 22 en lugar de 41 .

Para el caso más favorable, se ha considerado que habrá un aumento de demanda que depende del aumento de la población, pero la población general, la población mayor de 65 años, la cirugía mínimamente invasiva de próstata, el cáncer de vejiga, el cáncer de próstata, el cáncer de riñón, la hipertrofia benigna de próstata, la litiasis renal, la incontinencia urinaria, y la disfunción eréctil, contribuirán, cada uno de ellos, a una disminución del $2 \%$ de la demanda de aquí al año 2010. En este escenario, si se quisiera mantener el nivel de asistencia actual sobrarían 218 urólogos, y en consecuencia habría que formar cada año 10 urólogos en lugar de 41 .

Para el caso más desfavorable, se ha considerado que habrá un aumento de demanda que depende del aumento de la población, y además la población general, la población mayor de 65 años, la cirugía mínimamente invasiva de próstata, el cáncer de vejiga, el cáncer de próstata, el cáncer de riñón, la hipertrofia benigna de próstata, la litiasis renal, la incontinencia urinaria, y la disfunción eréctil, sufrirán algún tipo de cambios de tal manera que cada uno de ellos contribuirá a un aumento del $2 \%$ de la demanda de aquí al año 2010. En este escenario, si se quisieran mantener el nivel de asistencia actual sobrarían 40 urólogos, y en consecuencia habría que formar cada año 35 en lugar de 41. Sin embargo, si se quisieran mantener los ratios actuales de asistencia por habitante mayor de 65 años faltarian 43 urólogos, y en consecuencia habría que formar cada año 47 en lugar de 41 .

\section{DISCUSIÓN}

La falta de conocimiento preciso sobre la situación y previsiones de los recursos humanos en urología, llevaron a la AEU a formularse preguntas sobre el futuro de la Urología en España, especialmente sobre las necesidades en el número de urólogos que permita prestar asistencia sanitaria de calidad a la población española y de forma equitativa. El primer obstáculo para responder a estas preguntas es que no se conoce con precisión el número de urólogos en España, su distribución por edad, subespecialidad, o regiones, ni existen claros criterios para determinar el número de urólogos necesarios en un país determinado. Conocer el número de urólogos en cualquier país no es una tarea fácil. Por ejemplo, en los Estados Unidos (EE.UU) no se sabe con precisión el número de urólogos. En el año 2000, último año del que se disponen de cifras, había en los Estados Unidos 9.511 urólogos, según la Asociación Americana de Medicos (AMA). Sin embargo, en esa fecha, la Asociación de Urología Americana (AUA) tenía 8.300 miembros. Para precisar el número de urólogos, la AUA encargó una encuesta que identificó a 9.940 urólogos, es decir unos 500 más de los estimados por la AMA y unos 1.600 más de los miembros de la $\mathrm{AUA}^{31}$.

En Europa, el European Board of Urology realiza encuestas periódicas sobre el número de urólogos en determinados países europeos. La última encuesta, publicada en el año 2000, recoge 
información enviada por cada país con datos de $1998^{32}$. La variabilidad en el número de habitantes por urólogo es muy amplia. Mientras que algunos países, como Grecia, tienen un urólogo por cada 15.000 habitantes, otros, como Irlanda, tienen un urólogo por cada 180.000 habitantes. Según esos datos, España tiene 23.500 habitantes por urólogo, mientras que el Reino Unido tiene 130.000, Holanda 62.000, o Francia 58.500. Aunque estos datos son los mejores disponibles, deben ser tomados con cautela debido a los probables sesgos de infra o sobreestimación que pueden producirse debido a la metodología utilizada para recoger las estimaciones en los países individuales. Por otra parte, son datos de 1988 y en algunos países las cifras de urólogos por población podrían haber cambiado. Ante esta disparidad de cifras, la Asociación Europea de Urología, recomendaba, en el año 2002, la cifra de un urólogo por cada 45.000 habitantes. También esta recomendación puede tener matices en algunos países. Por ejemplo, en el Reino Unido, que es uno de los países con menos urólogos por población ${ }^{32}$, parte de la cirugía urológica es realizada por cirujanos generales.

Las formas de aproximarse al problema de conocer el número de urólogos y de estimar los urólogos necesarios para el futuro utilizadas en este estudio son muy novedosas, aportan información muy sólida, y presentan una gran flexibilidad que seguirá permitiendo estimar necesidades futuras según cambien las circunstancias, o mejore la información. El hecho de haber buscado los urólogos entre los socios de la AEU, suscriptores de revistas urológicas de ENE ediciones, miembros de servicios o unidades de urología de todos los hospitales españoles, y urólogos de las cinco compañías aseguradoras más importantes de toda España, hace pensar que el número de urólogos no detectados debe ser virtualmente nulo. Es decir, seguramente los 2.033 urólogos detectados es un número muy cercano al número real de urólogos activos en España. De estos urólogos, se conoce en todos la provincia en la que trabaja y el sexo, y en casi todos se conoce si trabajan en el sector público, privado o ambos. Otras variables, como la especialidad, la edad, o la categoría profesional se conoce en un número menor de urólogos por lo que se envió un cues- tionario administrado mediante encuesta postal de entre una y tres oleadas a los 2.033 urólogos identificados.

Por los hallazgos de este estudio, la población de urólogos es relativamente joven: la edad media de los urólogos españoles es de 46 años, y menos de un $20 \%$ tienen más de 55 años. La urología es una especialidad en la que predominan los hombres (92\%). Una gran mayoría de urólogos (83\%) trabaja en el sector público, algo más de la mitad (54\%) trabaja en el privado, y por lo tanto, uno de cada tres (37\%) urólogos trabaja en ambos sectores. La subespecialidad más citada de las que practican los urólogos españoles es la oncología, que declaran practicarla la mitad de los urólogos, mientras que sólo el 13\% hacen urología pediátrica. Naturalmente, las subespecialidades no son excluyentes, y de hecho más de la mitad de los urólogos practican más de una de las subespecialidades. El grueso de los urólogos tiene la categoría de médico adjunto, representando al $65 \%$ de los urólogos españoles, y algo más del $12 \%$ son residentes. Son miembros de la AEU el $80 \%$ de los urólogos españoles, y aunque esta cifra es alta, la AEU debería intentar atraer a ese $20 \%$ de urólogos activos que todavía no son miembros de la AEU.

La población española tiene un urólogo por cada 20.707 habitantes. Esta cifra de urólogos es alta comparada con otros países. De hecho, en Europa, sólo Grecia, Austria, o Luxemburgo, tienen más urólogos por población que España. En los Estados Unidos de Norteamérica hay también menos urólogos por población que en España (uno por cada 26.300 habitantes). Dentro de España, existe una gran variabilidad en el número de urólogos por población entre las diferentes CCAA. Por ejemplo, Navarra (13.998 habitantes por urólogo), tiene el doble de urólogos por población que Canarias (28.509 habitantes por urólogo). Las diferencias entre otras CCAA es también muy notable. El sistema sanitario español debería intentar corregir este tipo de variabilidad, dado que puede ser una de las fuentes de desigualdades en el acceso a las prestaciones asistenciales en urología.

Uno de los instrumentos desarrollados en este proyecto es el modelo predictivo. Este modelo permite estimar los urólogos disponibles y los 
que serán necesarios desde el año 2003 al año 2025, tanto para el conjunto nacional como para cada una de las 17 CCAA. El modelo predictivo funciona mediante la interacción de la información contenida o asumida en cuatro módulos: oferta, demanda, asunciones (sobre la oferta y la demanda) y criterios. La información de las variables de cada uno de los módulos puede ser cambiada por el usuario, de manera que constituye un instrumento muy útil por permitir construir numerosos escenarios que pueden darse ahora o en el futuro, y en una u otras regiones. La robustez metodológica del modelo radica en que contiene la base de datos de los 2.033 urólogos que hay en España, las cifras de población de aquí al año 2025 de España y de cada una de sus CCAA por tramos de edad y sexo, la carga de trabajo proporcional de las patologías urológicas más relevantes, y unas formulaciones matemáticas para aplicarlas bajo diferentes asunciones o criterios.

El modelo predice con precisión el número de urólogos que serán necesarios o que sobrarán en un año concreto o en una CCAA concreta bajo diferentes asunciones que construyen diferentes escenarios. Con los análisis de sensibilidad realizados con el modelo se estima que sólo será necesario aumentar el número de urólogos que estarán activos en España en escenarios futuros de gran aumento de demanda.

Este estudio, también tiene ciertas limitaciones. Quizá la limitación más importante es la tasa de respuesta a la encuesta dirigida a los urólogos, que aun siendo alta, no han respondido el $40 \%$. Para los datos fundamentales, como la provincia de residencia, o el sexo del urólogo, la tasa de respuesta no es una limitación, dado que se dispone de estos datos de todos los urólogos. En el caso del sector, se conoce si el urólogo trabaja en el sector público o privado en el $92 \%$ de los casos, lo cual tampoco es una limitación importante. Para los análisis descriptivos, tampoco es una limitación disponer de la edad del 78\% de los sujetos, pero sí lo podría ser para conocer la proporción de urólogos que estarán jubilados en un año determinado en el modelo predictivo. Para resolver esta limitación, el modelo asigna a los 457 urólogos cuya edad se desconoce, una edad obtenida de forma aleatoria de los 1.576 urólogos de los que sí se sabe la edad. Esta forma de resol- ver la falta de información de la edad se basa en la asunción de que la distribución de edades en los urólogos cuya edad se conoce es similar a la distribución de la edad en los urólogos cuya edad se desconoce. De esta manera el efecto que puede tener la ausencia de datos de edad en el $22 \%$ de la población de urólogos afectará de forma despreciable la precisión de las estimaciones para el conjunto nacional, y de forma mínima las estimaciones para cada CCAA. Para resolver este problema en futuros años, se deberían realizar periódicamente encuestas, especialmente centradas en los urólogos en los que exista poca información.

La metodología utilizada en este estudio, sus hallazgos, y las herramientas desarrolladas son un hito en la forma de abordar el arduo problema de predecir necesidades en recursos humanos de una especialidad concreta en un país determinado. Por lo tanto, los resultados de este estudio deberían ser difundidos entre interesados, mediante diversos medios, como publicaciones, o a través de la página web de la AEU. Este estudio puede ser muy útil para ayudar a la AEU y a sus asociaciones regionales a explorar con los responsables de la sanidad española, la formación médica de pregrado y postgrado en la planificación de recursos humanos en el área de la urología.

\section{CONCLUSIONES}

1. La metodología utilizada en este estudio permite conocer de forma bastante precisa el número y las características de los urólogos españoles.

2. El modelo predictivo desarrollado en este estudio es metodológicamente robusto $\mathrm{y}$ adaptable a escenarios en los que puedan producirse cambios en la oferta, demanda, asunciones, o criterios.

3. En España hay 2.033 urólogos activos en el año 2003, lo que significa que disponemos de un urólogo por cada 20.707 habitantes.

4. España tiene más urólogos por millón de habitantes que los Estados Unidos y que casi todos los países europeos.

5. En España, existe una enorme variabilidad en el número de urólogos por población: algunas CCAA, como Navarra, tienen el doble de urólogos por población que otras, como Canarias. 
6. La mayoría de los urólogos españoles son hombres, trabajan en el sector público, y practican varias subespecialidades urológicas.

7. Sólo en escenarios de gran aumento de demanda se necesitarían formar más de los 41 residentes que se vienen formando anualmente en España.

8. El 20\% de los urólogos españoles no son miembros de la AEU.

9. El esfuerzo de haber realizado este estudio debería mantenerse en el tiempo para tener datos actualizados y realizar mejores previsiones en el futuro.

10. Una de las formas de hacer que los hallazgos de este estudio sean útiles consiste en difundirlos, entre otros medios, en la página web de la AEU, y en utilizarlos para ayudar a los responsables políticos en la planificación sanitaria.

Agradecimientos: A Lola Sánchez Jódar, (secretaria de la AEU) por mantener y vehicular la información entre la AEU y el equipo investigador de TAISS, a ENE ediciones por su ayuda con los datos iniciales, A. Kathy Fitch por su contribución en el diseño inicial del proyecto y comentarios al manuscrito, a Ignacio Lázaro por su constancia en la búsqueda en Internet de los urólogos de las compañias aseguradoras privadas y por su brillante resolución de los aspectos matemáticos e informáticos del modelo predictivo, y a los urólogos que, respondiendo a la encuesta, han hecho posible este estudio.

\section{REFERENCIAS}

1. CHICHARRO-MOLERO JA, BURGOS-RODRÍGUEZ R, SÁNCHEZ-CRUZ JJ, DEL ROSAL-SAMANIEGO JM, RODERO-GARCÍA P, RODRÍGUEZ-VALLEJO JM.: Prevalence of benign prostatic hyperplasia in Spanish men 40 years old or older. Journal of Urology 1998; 159 (3): 878-882.

2. GÓMEZ ACEBO A, RODRÍGUEZ VALLEJO JM, RODRÍGUEZ MORA VI, GARCÍA ALCÁZAR I.: Quality of life and symptomatology in benign prostate hyperplasia in active Spanish population. Medicina Clinica 2000; 114 (3): 81-89.

3. PLATZ EA, KAWACHI I, RIMM EB, WILLETT WC, GIOVANNUCCI E.: Race, ethnicity and benign prostatic hyperplasia in the health professionals followup study. Journal of Urology 2000; 163 (2): 490-495.

4. SAGNIER PP, MACFARLANE G, RICHARD F, BOTTO H, TEILLAC P, BOYLE P.: Results of an epidemiological survey using a modified American Urology Asso- ciation symptom index for benign prostatic hyperplasia in France. Journal of Urology 1994; 151: 1266-1270.

5. TRUEMAN P, HOOD SC, NAYAK US, MRAZEK MF.: Prevalence of lower urinary tract symptoms and self-reported diagnosed "benign prostatic hyperplasia", and their effect on quality of life in a community-based survey of men in the UK. BJU International 1999; 83 (4): 410-415.

6. TSUKAMOTO T, KUMAMOTO Y, MASUMORI N et al.: Prevalence of prostatism in Japanese men in a community based study with comparison to a similar American study. Journal of Urology 1995; 154: 391-395.

7. ANSONG KS, LEWIS C, JENKINS P, BELL J.: Epidemiology of erectile dysfunction: a communitybased study in rural New York State. Annals of Epidemiology 2000; 10 (5): 293-296.

8. BLANKER MH, BOSCH JL, GROENEVELD FP, BOHNEN AM, PRINS A, THOMAS S, HOP WC.: Erectile and ejaculatory dysfunction in a community-based sample of men 50 to 78 years old: prevalence, concern, and relation to sexual activity. Urology 2001; 57 (4): 763-768.

9. Epidemiological Study of Erectile dysfunction in Thailand. An epidemiological study of erectile dysfunction in Thailand (Part 1: Prevalence). Thai Erectile Dysfunction Epidemiologic Study Group (TEDES). Journal of the Medical Association of Thailand 2000; 83 (8): 872-879.

10. FELDMAN HA, GOLDSTEIN I, HATZICHRISTOU DG et al.: Impotence and its medical and psychosocial correlates: results of the Massachussets Male Aging Study. Journal of Urology 1994; 151: 54-61.

11. GLASSER D, SWEENEY M.: The prevalence of erectile dysfunction in four countries: Italy, Brazil, Malaysia and Japan. Cross-national Study group. Presented at International consultation on erectile dysfunction. Paris, France 1999 july: 1-3.

12. KOSKIMAKI J, HAKAMA M, HUHTALA H, TAMMELA TL.: Effect of erectile dysfunction on frequency of intercourse: a population based prevalence study in Finland. Journal of Urology 2000; 164 (2): 367-370.

13. MARTÍN-MORALES A, SÁNCHEZ-CRUZ JJ, SÁENZ DE TEJADA I, RODRÍGUEZ-VELA L, JIMÉNEZCRUZ JF, BURGOS-RODRÍGUEZ R.: Prevalence and independent risk factors for erectile dysfunction in Spain: results of the "Epidemiología de la Disfunción Eréctil Masculina Study." Journal of Urology 2001; 166 (2): 569-574.

14. MARUMO K, NAKASHIMA J, MURAI M.: Age-related prevalence of erectile dysfunction in Japan: assessment by the International Index of Erectile Function. International Journal of Urology 2001; 8 (2): 53-59.

15. MAGGI S, MINICUCI N, LANGLOIS J, PAVAN M, ENZI G, CREPALDI G.: Prevalence rate of urinary incontinence in community-dwelling elderly individuals: the Veneto study. Journals of Gerontology Series A-Biological Sciences \& Medical Sciences 2001; 56 (1): M14-M18. 
16. GAVIRA IGLESIAS FJ, CARIDAD Y OCERIN JM, PÉREZ DEL MOLINO MARTÍN J, VALDERRAMA GAMA E, LÓPEZ PÉREZ M, ROMERO LÓPEZ M, PAVÓN ARANGUREN MV, GUERRERO MUÑOZ JB.: Prevalence and psychosocial impact of urinary incontinence in older people of a Spanish rural population. Journals of Gerontology Series ABiological Sciences \& Medical Sciences 2000; 55 (4): M207-M214.

17. SMOGER SH, FELICE TL, KLOECKER GH.: Urinary incontinence among male veterans receiving care in primary care clinics. Annals of Internal Medicine 2000; 132 (7): 547-551.

18. SIMEONOVA Z, MILSOM I, KULLENDORFF AM, MOLANDER U, BENGTSSON C.: The prevalence of urinary incontinence and its influence on the quality of life in women from an urban Swedish population. Acta Obstetricia et Gynecologica Scandinavica 1999; 78 (6): 546-551.

19. SAMUELSSON E, VÍCTOR A, SVARDSUDD K.: Determinants of urinary incontinence in a population of young and middle-aged women. Acta Obstetricia et Gynecologica Scandinavica 2000; 79 (3): 208-215.

20. ZUNZUNEGUI MV, RODRÍGUEZ-LASO A, GARCÍA DE YÉBENES MJ, AGUILAR MD, LÁZARO P, OTERO A.: Prevalencia de incontinencia urinaria y factores asociados en hombres y mujeres de más de 65 años. Atención primaria (en prensa, 2003).

21. CURHAN GC, WILLETT WC, RIMM EB, SPEIZER FE, STAMPFER MJ.: Body size and risk of kidney stones. Journal of the American Society of Nephrology 1998; 9: 1645-1652.

22. GRASES F, CONTE A, MARCH JG, GENESTAR C, COSTA-BAUZA A, MARTÍN M, VALLESCAR R.: Epidemiology of urinary stone disease in the Balearic Islands Community. International Urology \& Nephrology 1994; 26: 145-150.

23. HIATT RA, DALES LG, FRIEDMAN G, HUNKELER EM.: Frequency of urolithiasis in a prepaid medical care program. American Journal of Epidemiology 1982; 115: 255-265.

24. SERIO A, FRAIOLI A.: Epidemiology of nephrolithiasis. Nephron 1999; Suppl 1: 26-30.
25. SIMON J, HUDES ES.: Relation of serum ascorbic acid to serum vitamin B12 and serum ferritin and kidney stones in the US. Archives Internal Medicine 1999; 159: 619-624.

26. SOUCIE JM, COATES RJ, MCCLELLAN W, AUSTIN H, THUN M.: Relation between geographic variability in kidney stones prevalence and risk factors for stones. American Journal of Epidemiology 1996; 143 (5): 487-495.

27. SOWERS MR, JANNAUSCH M, WOOD C, POPE SK, LACHANCE LL, PETERSON B.: Prevalence of renal stones in a population-based study with dietary calcium, oxalate, and medication exposures. American Journal of Epidemiology 1998; 147 (10): 914-920.

28. TRINCHIERI A, COPPI F, MONTANARI E, DEL NERO A, ZANETTI G, PISANI E.: Increase in the prevalence of symptomatic upper urinary tract stones during the last ten years. European Urology 2000; 37: 23-25.

29. CURHAN GC, WILLET WC, RIMM EB, SPIEGELMAN D, STAMPFER MJ.: Prospective study of beverage use and the risk of kidney stones. American Journal of Epidemiology 1996; 143: 240-247.

30. Cáncer: incidencia y mortalidad en España, año 1997. A partir de todos los registros de cáncer de España en la European Networks of Cancer Registries. IARC, Lyon 1999.

31. American Urologic Association. [Internet] www.auanet.org. Visitada: 7/Feb/2003.

32. KIELY EA.: The European Board of Urology survey of current urological manpower, training and practice in Europe. BJU International 2000; 85: 8-13.

Dr. P. Lázaro y de Mercado

Técnicas Avanzadas de Investigación

en Servicios de Salud (TAISS)

C/ Cambrils, $41-2$

28034 Madrid

e-mail: plazaro@tais.com

(Trabajo recibido el 7 julio de 2003) 